\title{
Hyperphosphorylation of Tau Protein in Hippocampus of Central Insulin- Resistant Rats is Associated with Cognitive Impairment
}

\author{
Dantao Penga,e Xiuqing Pan ${ }^{\mathrm{b}, \mathrm{e}}$ Ju Cuic Yueqin Ren ${ }^{\mathrm{d}}$ Jiewen Zhang ${ }^{\mathrm{b}}$ \\ aDepartment of Neurology, Beijing Hospital, Ministry of Health, Beijing, 'Department of Neurology, \\ Zhengzhou First People's Hospital, Zhengzhou, 'Beijing Institute of Geriatrics, Beijing Hospital, Ministry \\ of Health, Beijing, dThe Second Affiliated Hospital of Henan Traditional Chinese Medicine University, \\ Zhengzhou, China; ${ }^{\text {EE}}$ Equally contributed to this work
}

\section{Key Words}

Insulin resistance $\cdot$ Tau protein • Alzheimer's disease • Streptozotocin • Hyperphosphorylation

\begin{abstract}
Background: Alzheimer's disease (AD) is one of the most common neurodegenerative diseases. Peripheral insulin resistance increases the risk for memory impairment and the development of AD. Objective: This study aims to assess changes in cognitive functions and the level of hyperphosphorylated tau proteins in central insulin-resistant rats. Methods: An in vivo central insulin-resistant (CIR) animal model was generated through intracerebroventricular injection of streptozotocin (STZ) into insulin-resistant (IR) rats that were induced by feeding a highglucose/-protein/-fat diet. The Morris water maze test was used to assess changes in cognitive functions, pathological changes in the cornu ammonis 1 (CA1) region of the hippocampus were detected by immunohistochemistry, and the phosphorylation levels of tau proteins at specific sites were determined by Western blot analysis. Results: The escape latency time in the Morris water maze test was significantly prolonged; the number of phosphorylated tau proteins in the CA1 region of the hippocampus was significantly increased; and the phosphorylation levels of tau proteins at Ser199, Thr205, Thr212, Thr217 and Ser396 were significantly elevated in the CIR group compared with the IR and control groups. Conclusion: This study provides direct evidence that CIR plays an important role in AD pathogenesis by facilitating tau hyperphosphorylation.
\end{abstract}

Dantao Peng and Jiewen Zhang
Department of Neurology, Beijing Hospital, Ministry of Health, Beijing (China) and Department of Neurology, Zhengzhou First People's Hospital, Zhengzhou (China)

E-Mail pengdantao@medmail.com.cn and E-Mail zhangjiewen9900@126.com 


\section{Introduction}

Alzheimer's disease (AD) is one of the most common neurodegenerative diseases, and is characterized by progressive functional disturbances in cognition and memory. The well-established core pathological features of AD include accumulation of intracellular neurofibrillary tangles (NFTs) [1] and extracellular deposits of senile plaques (SPs) [2, 3] in the hippocampus and cortex. However, the exact etiology and pathogenesis of AD remain unclear. Epidemiological studies have shown that the prevalence of AD in people aged over 65 years is about $2-5 \%$, and this rate doubles with every 5 -year increment in age after 65 to reach $47 \%$ in those aged 85 and over [4]. The mortality of AD also increases with advancing age [5]. Therefore, the ever-expanding population of $\mathrm{AD}$ patients is of great social and economic concerns.

Although age remains the main risk factor for the development of $\mathrm{AD}$, the underlying mechanisms are not completely understood. Multiple studies have demonstrated that tau protein is the major protein component of NFTs in the brain of AD patients, and abnormally hyperphosphorylation of tau protein plays a key role in NFT formation [6]. Accumulating evidence indicates that insulin resistance (IR) is one of the risk factors for $\mathrm{AD}$, and abnormal insulin levels in the brain may be an important cause of AD [7-11]. Streptozotocin (STZ) is a glucosamine-nitrosourea compound that decreases both insulin receptor autophosphorylation in neurons and endogenous tyrosine kinases activation [12, 13], which may increase the activity of phosphotyrosine phosphatase to inhibit insulin signaling [14]. In recent years, intracerebroventricular injection of STZ has been employed by some investigators to generate a rat model of AD by means of inducing central insulin resistance (CIR) [15-17]. In this study, the underlying molecular mechanisms of CIR in the development of $\mathrm{AD}$ were investigated with an in vivo animal model generated through intracerebroventricular injection of STZ into IR rats. T We analyzed changes in rat cognitive function and tau phosphorylation levels. Our results elucidate how abnormalities in insulin signaling can facilitate AD pathogenesis.

\section{Materials and Methods}

\section{Reagents and materials}

STZ and an anti-actin antibody were obtained from Sigma-Aldrich (St. Louis, MO, USA). Anti-phosphoTau (Ser199, Thr 205, Thr 212, Thr 217, Ser 396) antibodies were from Bio-Source International (Camarillo, CA, USA). The monoclonal anti-tau antibody AT-100 (specific for phospho-Ser212 and -Thr214) was from Innogenetics (Ghent, Belgium). The polyclonal anti-Iba1 antibody was from Wako Chemicals (Richmond, VA, USA).

\section{Animals}

Thirty 4-week-old, healthy male Wistar rats of specific pathogen-free (SPF) grade, weighing 120-150 g, were provided by the Experimental Animal Center of Zhengzhou University (Certificate No.: SCXK-Henan 2010-0002). Mouse experimentation was carried out in strict accordance with the recommendations in the guide for the Care and Use of Laboratory Animals of the United States National Institutes of Health. The protocol was approved by the Institutional Animal Care and Application Committee of the Beijing Institute of Geriatrics (approval no. 20101017). After 1-week adaptive feeding, the rats were randomly divided into three groups ( $\mathrm{n}=10$ in each group): a normal control (NC) group, an IR group, and a CIR group. Rats in the NC group were fed a normal diet, while those in the IR and CIR groups were fed a high-glucose, highprotein, and high-fat diet (calorie percentages: carbohydrates 26\%, proteins $15.2 \%$, fats [lard)] 58.8\%). After 3 months, fasting plasma insulin (FPI) and fasting blood glucose (FBG) levels were measured, and homeostatic model assessment was performed to determine the IR index (HOMA-IR) were calculated. Model success was demonstrated by a significant difference in HOMA-IR between the NC and IR/CIR groups. After IR induction, animals in the CIR group underwent an intracerebroventricular bolus injection of STZ (dissolved in 0.1mol/L citrate buffer, pH 4.3) at a dose of 3.0-3.5 mg/kg, which can interfere with 
Peng et al.: Cognitive Impairment and Phosphorylation of Tau Protein in Insulin Resistance Rats

neuronal insulin signaling pathways. Following the methods outlined by Sharma et al. [18], the rats were anesthetized with an intraperitoneal injection of $10 \%$ chloral hydrate $(0.35 \mathrm{~g} / \mathrm{kg})$ and fixed on a stereotaxic instrument. After conventional fur removal and sterilization, the scalp skin was cut open to expose the cranial bone. A dental drill was used to make an opening $1.5 \mathrm{~mm}$ behind the anterior fontanel and $1.5 \mathrm{~mm}$ on the left and right of interparietal suture to open and expose the endocranium [19]. A microinjector was inserted vertically $3.5 \mathrm{~mm}$ below the cerebral surface. Meanwhile, rats in the NC and IR groups were injected intracerebroventricularly injected with the same dose of citrate buffer according to the method described above. During the experiments, all rats were raised in clean-grade animal chambers at constant temperature $\left(23^{\circ} \mathrm{C}\right)$ with a 12 -hour light/dark cycle.

\section{FBG and FPI measurement}

Blood samples were collected from the tail veins of rats after a 12-hour fast. FBG concentration was measured with a OneTouch glucosemeter (Johnson Inc.). Prior to sacrifice, $1 \mathrm{ml}$ blood was collected from the heart under fasting conditions. Plasma was collected after centrifugation of the blood sample. FPI levels were measured using a radioimmunoassay kit (Beijing Atomic Energy Research Laboratory). HOMA-IR was calculated using the following formula: HOMA-IR=FPIs (IU/L)×FBG (mmol/L)/22.5 [20].

\section{Morris water maze test}

A modified Morris water maze [21] test was used to evaluate changes in rat cognitive functions. Experiments commenced 16 days after intracerebroventricular injection of STZ or citrate buffer. The maze consists of a round pool (black interior walls and floors; diameter, $150 \mathrm{~cm}$; height, $50 \mathrm{~cm}$ ) filled with 30$\mathrm{cm}$ water that was made opaque with black ink. The pool was divided into four equally spaced quadrants, and a round platform (diameter, $10 \mathrm{~cm}$; height, $29 \mathrm{~cm}$ ) was placed in the middle of one quadrant, $1.0 \mathrm{~cm}$ below the water surface. The temperature of the water was maintained at $20-23^{\circ} \mathrm{C}$. All experiments were carried out in a quiet room between 10:00 am and 4:00 pm by the same experimenter. The water in the pool was changed every day, and the rats were subjected to 4-hour fasting before each experiment. Testing was conducted in two phases: acquisition and retention. Animals were placed at the same starting point in each of the experiments. Initially, all animals were trained for 5 consecutive days with two trials/day. The time limit was $120 \mathrm{~s} /$ trial with an intertribal interval of $5 \mathrm{~min}$. If the animal failed to find the platform, it was manually placed on it for $10 \mathrm{~s}$. The retention phase consisted of a single trial that started 1 day after the acquisition phase was completed. The escape latency time (defined as the time taken by the animal to locate the escape platform) in both phases was recorded.

\section{Western blot and immunohistochemistry}

Hippocampal tissue was dissected and lysed in $50 \mu \mathrm{l}$ RIPA buffer, followed by centrifugation at $13,000 \mathrm{rpm}$ for $15 \mathrm{~min}$ at $4^{\mathrm{o}} \mathrm{C}$. Supernatants were collected for sodium dodecyl sulfate-polyacrylamide gel electrophoresis (SDS-PAGE) and Western blot analysis. The samples (30 $\mu$ g protein, as determined with a Bio-Rad assay kit) were separated by SDS-PAGE and transferred to PVDF membranes, blocked by 5\% milk in Tris-buffered saline with Tween (TBST) and blotted with anti-phospho-Tau (p-Ser199-Tau, 1:1000), anti-phospho-Tau (p-Thr205-Tau, 1:1000), anti-phospho-Tau (p-Thr212-Tau, 1:1000), anti-phosphoTau (p-Thr217-Tau, 1:1000), anti-phospho-Tau (p-Ser396-Tau, 1:1000), or anti-actin (1:2000) primary antibodies. An enhanced chemiluminescence kit (Pierce) was used to detect immunoreactive bands. The Image Image J software (National Institutes of Health, Bethesda, MD, USA) was used for calibration and semi-quantitative analysis of the optical density values of the bands from different groups.

Paraffin sections were blocked with Peroxidase-Blocking Reagent (Dako) for $10 \mathrm{~min}$ and then incubated with blocking solution (5\% goat serum, 3\% bovine serum albumin [BSA] and 0.5\% triton X-100 in phosphate-buffered saline [PBS]) for 1 hour at room temperature. Afterwards, specimens were incubated with anti-tau antibody AT-100 (1:200) or anti-Iba1 antibody (1:500) at $4^{\circ} \mathrm{C}$ overnight. After being washed with TBST, the sections were incubated with horseradish peroxidase (HRP)-conjugated anti-mouse or anti-rabbit secondary antibodies at room temperature for 1 hour. Visualization of insulin or Kif5b staining was performed using liquid DAB (3,3'-diaminobenzidine) and substrate-chromogen solution from the EnVision+ System-HRP (DAB) (Dako). Three sections from each group, collected from the same site, were observed under a light microscope (Carl Zeiss) and photographed using Microphoto-FX (Nikon), a digital camera (Nikon), and SPOT Advanced imaging software. The images were analyzed using ImageJ software to 
Table 1. Comparisons of fasting blood glucose (FBG), fasting plasma insulin (FPI) and insulin resistance index (HOMA-IR) levels among control (NC), insulin resistance (IR) and central insulin resistance (CIR) rats. Significant increase in FBG, FPI and HOMA-IR levels were detected in IR and CIR rats comparing with CT rats $\left(* P<0.05,{ }^{* *} P<0.01\right.$.). Compared with IR rats, CIR rats had higher levels of FBG, FPI and HOMA-IR $(\Delta<0.05)$. Data are expressed as mean \pm S.D. and are representative of 10 rats. Differences were detected with one-way ANOVA followed by Tukey's test

\begin{tabular}{lccc}
\hline Groups & NC & IR & CIR \\
\hline Diet & Normal diet & High-glucose & High-glucose \\
& & High-protein & High-protein \\
Icv. STZ injection & No & High-fat diet & Yes \\
FBG (mmol/L) & $4.14 \pm 0.72$ & $6.33 \pm 0.98^{*}$ & $8.05 \pm 0.89^{* *} \Delta$ \\
FPI (IU/L) & $63.14 \pm 12.20$ & $92.60 \pm 9.14^{*}$ & $135.57 \pm 18.68^{* *} \Delta$ \\
HOMA-IR & $11.84 \pm 4.16$ & $26.21 \pm 5.68^{*}$ & $48.87 \pm 10.87^{* *} \Delta$ \\
\hline
\end{tabular}

determine the mean optical density values of positive-labeled areas in the cornu ammonis 1 (CA1) region of the hippocampus.

\section{Statistical analysis}

SPSS 11.5 was used to analyze all data which were expressed as mean \pm S.D. For multiple comparisons of data, the significant differences were determined by one-way or two-way analysis of variance (ANOVA) as indicated. $\mathrm{P}<0.05$ was considered to be statistically significant.

\section{Results}

\section{IR and CIR rat model generation}

A high-glucose, high-protein and high-fat diet was used to induce IR in rats. After induction of IR, STZ was intracerebroventricularly injected to generate CIR rats. The success of IR and CIR rat models was evaluated by measuring FBG and FPI levels. Both the FBG and FPI levels of the IR and CIR groups were significantly higher than those of the NC group; and the differences between the IR group and the CIR group were statistically significant $(P<0.05)$. The HOMA-IR index values in the IR and CIR groups were significantly higher than that of the NC group. Compared with the IR group, the HOMA-IR index in the CIR group was more significant $(P<0.05)$ (Table 1$)$.

\section{Cognitive function was significantly impaired in the IR and CIR groups}

The Morris water maze test was used to evaluate the spatial learning and memory abilities of rats in the IR and CIR groups. Two-way ANOVA (group $\times$ day) was used to detect differences among groups in the time to locate the hidden platform during the acquisition phase. The CIR rats showed significant impairment in locating the platform during the acquisition phase $\left(F_{(8,735)}=3.465, P<0.01\right)$. Daily analysis was performed with one-way ANOVA followed by Tukey's test showed that the escape latency times in the IR and CIR groups were significantly prolonged compared with the NC group $\left(* P<0.05\right.$, ${ }^{* *} P<0.01$, vs. control respectively). The escape latency for CIR rats was not significantly different from that of the IR group on the first day of training $\left(F_{(1,18)}=0.165, P=0.126\right)$; however, it was significantly higher than IR group values on the following training days. A significant difference in time to locate the hidden platform was observed in the CIR group on the first day of training compared with subsequent training days $\left(F_{(4,45)}=2.563, P<0.01\right)$. There was 
A

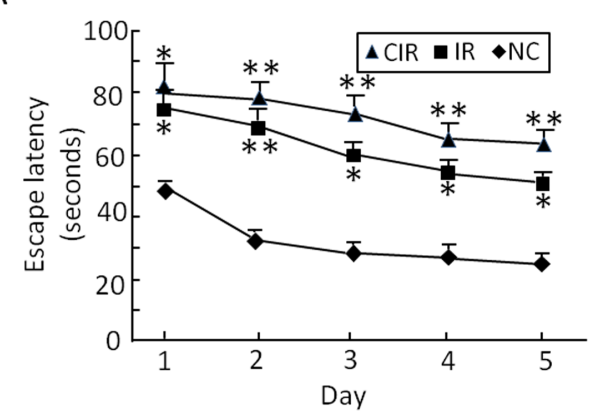

B

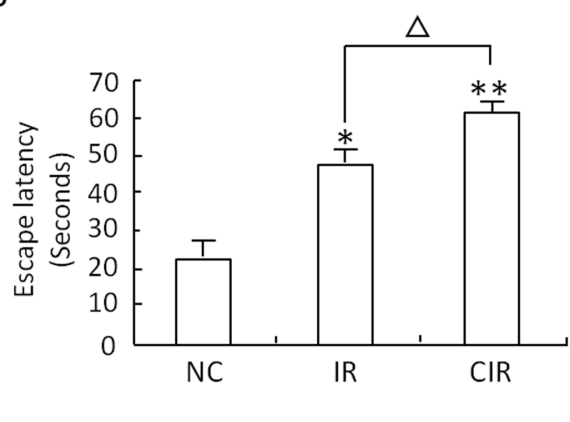

Fig. 1. The escape latency time in Morris water maze test of the rats. Compared with NC rats, the abilities of IR and CIR rats to escape from Morris water maze were significantly impaired in both acquisition phase (A) and retention phase (B). The escape latency time of the CIR rats were significantly prolonged than IR rats in both phases. Data expressed as mean \pm S.D. $(n=10)$. ${ }^{*} \mathrm{P}<0.05,{ }^{* *} \mathrm{P}<0.01$ vs. NC group, $\triangle \mathrm{P}<0.05$ vs. IR group were considered significant.

no significant learning impairment of rats in the $\operatorname{CIR}\left(F_{(4,45)}=0.275, P=0.843\right)$ or $\operatorname{IR}\left(F_{(4,45)}\right.$ $=0.375, P=0.724)$ groups concerning individual training days (Fig. $1 \mathrm{~A})$. The time to locate the hidden platform in the retention phase was analyzed by one-way ANOVA followed by Tukey's test, which showed that CIR rats exhibited significant memory impairment $\left(F_{(2,27)}\right.$ $=4.512, P<0.01$ vs. other groups) (Fig. 1B).

Tau hyperphosphorylation and microglial activation in the brains of IR and CIR rats

Immunohistochemistry analysis using anti-phospho-tau antibodies (AT-100) demonstrated the site-specific tau phosphorylation at Ser-212 and Thr-214 in the CA1 region of IR and CIR rats. The immunologically positive cells in the hippocampus were mainly distributed in the perinuclear region, and a large amount of positive buffy particles were present in the cytosol. Compared with the NC group, positive labeling in the same brain regions of the IR and CIR groups were significantly increased, and only a very small number of buffy particles were observed in the cytosol of the NC group (Fig. 2A-C). Moreover, the mean optical density values and number of neurons showing Tau phosphorylation in the CA1 region of IR and CIR rats were significantly higher than the NC group, indicating higher levels of phosphorylated tau proteins (Ser212 and Thr-214) (Fig. 2D, E). To assess neuroinflammation, we measured microglial activation by analyzing Iba1 expression levels. Immunostaining revealed greater expression of Iba1 in the CA1 region of rats in the IR and CIR groups (Fig. 2F-H). Quantitative analysis revealed that compared to the control group, neuroinflammation as assessed by microglial activation was increased in the CA1 of the IR and CIR rats (Fig. 2I, J).

Western blot analysis demonstrated that phosphorylation levels of tau proteins at Ser199, Thr205, Thr212, Thr217 and Ser396 sites in the hippocampi of IR and CIR rats were significantly higher than those of the NC group $(\mathrm{P}<0.05)$ (Fig. 3). Furthermore, the phosphorylation of tau at Ser199, Thr217 and Ser396 in the hippocampi of CIR rats was significantly higher than those in the IR group (Fig. 3).

\section{Discussion}

Insulin resistance refers to a decreased ability of insulin to maintaining normal blood glucose levels, that is, when a certain concentration of insulin cannot achieve the anticipated physiological effects or when tissue responses to insulin decrease. The reduction in insulin biopotency results in relatively insufficient insulin [22]. Notably, the relative deficiency of insulin is closely related with cognitive function impairment in $\mathrm{AD}$ patients [8-10]. AD is 
Peng et al.: Cognitive Impairment and Phosphorylation of Tau Protein in Insulin Resistance Rats

Fig. 2. (A-C) Immunohistochemistry of of tau proteins (AT-100) in the hippocampal CA1 region of the rats from NC, IR and CIR groups. Scale bar $=400$ um. (D, E) The mean optical density values and number of neurons with Tau phosphorylation in the CA1 region of IR and CIR rats were significantly higher than that of NC rats, indicating higher levels of phosphorylated tau proteins. Bars represent the mean \pm S.D. $(n=5)$. ${ }^{*} \mathrm{P}<0.05,{ }^{* *} \mathrm{P}<0.01$ vs. NC group were considered significant. (F-H) Microglial infiltration was revealed by immunostaining of Iba-1 in the CA1 region of the rats from NC, IR and CIR groups. Scale bar $=400$ um. (I, J) Quantative analysis of the mean optical density values and number of active microglial cells in the CA1 region reveals microglial activation in the CA1 region of IR and CIR rats. Bars represent the mean \pm S.D. $(n=5) .{ }^{*} \mathrm{P}<0.05$, $* * \mathrm{P}<0.01$ vs. NC group were considered significant.
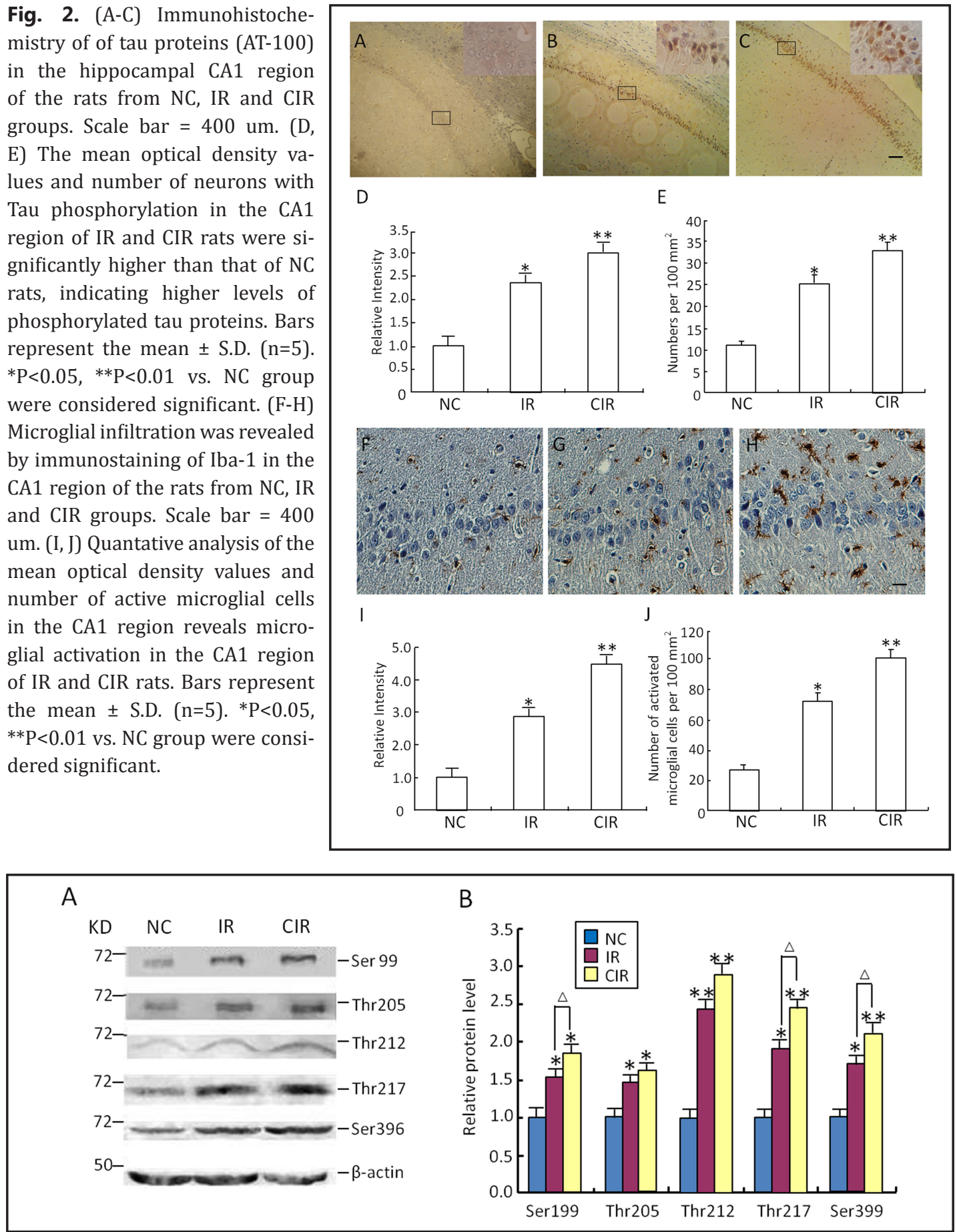

Fig. 3. Phosphorylation levels of tau proteins at Ser199, Thr205, Thr212, Thr217 and Ser396 in the hippocampi of the rats from each group. (A) Tau Proteins from hippocampus were separated by SDS-PAGE and identified with the antibodies listed on the left of each blot. (B) Semi-quantitative comparison of the phosphorylation levels of tau proteins at Ser199, Thr205, Thr212, Thr217 and Ser396 in the hippocampi of the rats from each group. Bars represent the mean \pm S.D. $(n=5) .{ }^{*} \mathrm{P}<0.05,{ }^{* *} \mathrm{P}<0.01$ vs. NC group, $\triangle \mathrm{P}<0.05$ vs. IR group were considered significant.

characterized by progressive memory impairment and cognitive function disturbance. Previous studies have revealed that impairment in the insulin/IR signaling system is a major reason for glucose/energy metabolism abnormalities in the brains of AD patients [23-25]. The 
present study generated the IR rat model by feeding a high-glucose, high-fat and high-protein diet for 3 months. After IR induction, rats in the CIR group underwent intracerebroventricular injection of STZ to establish an animal model with partial characteristics of AD. Findings from the Morris water maze test showed that compared with the NC group, learning and memory functions of rats in the IR and CIR groups were significantly decreased, and the escape latency time was obviously prolonged, indicating impaired cognitive functions. These changes were more obvious in the CIR group than in the IR group, indicating that CIR accelerated the progression of AD.

The typical pathological changes of AD include progressive losses of neurons and synapses, NFTs due to tau hyperphosphorylation, and extracellular deposits of amyloid- $\beta$ $(A \beta)$ as SPs [1-3]. Tau protein is a predominantly neuronal microtubule-associated protein that is enriched in axons and is capable of promoting microtubule assembly and stabilization [26]. Tau hyperphosphorylation is considered as one of the typical pathological changes in AD [27-29]. Hyperphosphorylated tau deposits in neuronal cells, leading to neuronal injury and degeneration, which facilitates neuronal apoptosis and accelerates the pathological changes of AD.

Tau phosphorylation is regulated by protein kinases and phosphatases: glycogen

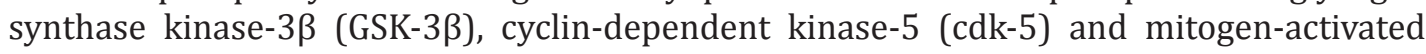
protein kinase (MAPK) can all promote tau phosphorylation [30]. Previous studies revealed that insulin could inhibit GSK-3 $\beta$ function via the phosphoinositide 3-kinase-protein kinase $\mathrm{B}$ (PI3K-PKB)/Akt pathway, which decreases tau phosphorylation [31]. In insulin gene knockout mice, insulin deficiency leads to an increase in GSK-3 $\beta$ activity accompanied by tau hyperphosphorylation [32]. In addition, the glycosylation of $\mathrm{N}$-acetylglucosamine (GluNAc) is also involved in regulating tau protein phosphorylation/dephosphorylation [33]. GluNAc can modify many kinds of microtubule-associated proteins and antagonize phosphorylation at multiple tau protein sites. Glycometabolism disturbance in the brain may increase tau phosphorylation by decreasing the concentration of GluNAc and thus facilitate the development of $\mathrm{AD}$ [33]. At least 12 tau-Ser and tau-Thr phosphorylation sites have been identified in the brains of AD patients, and we selected 5 to investigate the relationship between tau protein phosphorylation and insulin resistance (Ser199, Thr205, Thr212, Thr217 and Ser396). Different extents of hyperphosphorylation were observed at these sites in the IR and CIR rats but not the NC group, and the phosphorylation level in the CIR group was even higher than that in the IR group. These results suggest that impairments in neuronal signaling pathways lead to tau hyperphosphorylation and aggravate AD-like changes, supporting that insulin resistance is an important factor in the pathogenesis of AD [8-10].

In general, the present study showed that central insulin resistance induced by intracerebroventricularl injection of STZ into insulin-resistant rats leads to impairment of cognitive function, increased hyperphosphorylation of tau protein and microglial activation in the hippocampus, which shares similar pathological changes as AD. This indicates that central insulin resistance is closely associated with the pathogenesis and development of AD.

\section{Acknowledgements}

This work was supported by a grant from National Natural Science Foundation of China to D. P. (Project no.30971000). The authors declare that no conflict of interest existed.

\section{References}

1 Grundke-Iqbal I, Iqbal K, Tung YC, Quinlan M, Wisniewski HM, Binder LI: Abnormal phosphorylation of the microtubule-associated protein tau (tau) in Alzheimer cytoskeletal pathology. Proc Natl Acad Sci USA 1986;83:4913-4917. 
2 Glenner GG, Wong CW: Alzheimer's disease: initial report of the purification and characterization of a novel cerebrovascular amyloid protein. Biochem Biophys Res Commun 1984;120:885-890.

- 3 Glenner GG, Wong CW, Quaranta V, Eanes ED: The amyloid deposits in Alzheimer's disease: their nature and pathogenesis. Appl Pathol 1984;2:357-369.

4 Cummings JL, Cole G: Alzheimer disease. JAMA 2002;287:2335-2338.

5 Anderson RN, Smith BL: Deaths: leading causes for 2001. Natl Vital Stat Rep 2003;52:1-85.

6 Gong CX, Liu F, Grundke-Iqbal I, Iqbal K: Post-translational modifications of tau protein in Alzheimer's disease. J Neural Transm 2005;112:813-838.

7 Iqbal K, Alonso Adel C, Chen S, Chohan MO, El-Akkad E, Gong CX, Khatoon S, Li B, Liu F, Rahman A, Tanimukai H, Grundke-IqbaI I: Tau pathology in Alzheimer disease and other tauopathies. Biochim Biophys Acta 2005;1739:198-210.

8 Ott A, Stolk RP, Hofman A, van Harskamp F, Grobbee DE, Breteler MM: Association of diabetes mellitus and dementia: the Rotterdam Study. Diabetologia 1996;39:1392-1397.

-9 Luchsinger JA, Tang MX, Shea S, Mayeux R: Hyperinsulinemia and risk of Alzheimer disease. Neurology 2004;63:1187-1192.

10 Plastino M, Fava A, Pirritano D, Cotronei P, Sacco N, Sperlì T, Spanò A, Gallo D, Mungari P, Consoli D, Bosco D: Effects of insulinic therapy on cognitive impairment in patients with Alzheimer disease and diabetes mellitus type-2. J Neurol Sci 2010;288:112-116.

11 Ramos-Rodriguez JJ, Ortiz O, Jimenez-Palomares M, Kay KR, Berrocoso E, Murillo-Carretero MI, Perdomo G, Spires-Jones T, Cozar-Castellano I, Lechuga-Sancho AM, Garcia-Alloza M: Differential central pathology and cognitive impairment in pre-diabetic and diabetic mice. Psychoneuroendocrinology 2013;38:2462-2475.

12 Ar'Rajab A, Ahren B: Long-term diabetogenic effect of streptozotocin in rats. Pancreas 1993;8:50-57.

13 Park SW, Choi SA, Yun JW, Choi JW: Alterations in pancreatic protein expression in STZ-induced diabetic rats and genetically diabetic mice in response to treatment with hypoglycemic dipeptide Cyclo (His-Pro). Cell Physiol Biochem 2012;29:603-616.

14 Begum N, Sussman KE, Draznin B: Differential effects of diabetes on adipocyte and liver phosphotyrosine and phosphoserine phosphatase activities. Diabetes 1991;40:1620-1629.

- 15 Sonkusare S, Srinivasan K, Kaul C, Ramarao P: Effect of donepezil and lercanidipine on memory impairment induced by intracerebroventricular streptozotocin in rats. Life Sci 2005;77:1-14.

16 Kosaraju J, Gali CC, Khatwal RB, Dubala A, Chinni S, Holsinger RM, Madhunapantula VS, Muthureddy Nataraj SK, Basavan D: Saxagliptin: a dipeptidyl peptidase-4 inhibitor ameliorates streptozotocin induced Alzheimer's disease. Neuropharmacology 2013;72:291-300.

17 Lester-Coll N, Rivera EJ, Soscia SJ, Doiron K, Wands JR, de la Monte SM: Intracerebral streptozotocin model of type 3 diabetes: relevance to sporadic Alzheimer's disease. J Alzheimers Dis 2006;9:13-33.

18 Shoham S, Bejar C, Kovalev E, Weinstock M: Intracerebroventricular injection of streptozotocin causes neurotoxicity to myelin that contributes to spatial memory deficits in rats. Exp Neurol 2003;184:10431052.

19 Bao BM, Shu SY: Cerebral Stereotaxic Atlas of Rats. Beijing, People's Medical Publishing House, 1991, pp 12-18.

20 Hosker JP, Matthews DR, Rudenski AS, Burnett MA, Darling P, Bown EG, Turner RC: Continuous infusion of glucose with model assessment: measurement of insulin resistance and beta-cell function in man. Diabetologia 1985;28:401-411.

21 Morris R: Developments of a water-maze procedure for studying spatial learning in the rat. J Neurosci Methods 1984;11:47-60.

22 Kraegen EW, Clark PW, Jenkins AB, Daley EA, Chisholm DJ, Storlien LH: Development of muscle insulin resistance after liver insulin resistance in high-fat-fed rats. Diabetes 1991;40:1397-1403.

-23 Frolich L, Blum-Degen D, Bernstein HG, Engelsberger S, Humrich J, Laufer S, Muschner D, Thalheimer A, Türk A, Hoyer S, Zöchling R,Boissl KW, Jellinger K, Riederer P: Brain insulin and insulin receptors in aging and sporadic Alzheimer's disease. J Neural Transm 1998;105:423-438.

24 Talbot K, Wang HY, Kazi H, Han LY, Bakshi KP, Stucky A, Fuino RL, Kawaguchi KR, Samoyedny AJ, Wilson RS, Arvanitakis Z, Schneider JA, Wolf BA, Bennett DA, Trojanowski JQ, Arnold SE: Demonstrated brain insulin resistance in Alzheimer's disease patients is associated with IGF-1 resistance, IRS-1 dysregulation, and cognitive decline. J Clin Invest 2012;122:1316-1338. 
25 Schubert M, Brazil DP, Burks DJ, Kushner JA, Ye J, Flint CL, Farhang-Fallah J, Dikkes P, Warot XM, Rio C, Corfas G, White MF: Insulin receptor substrate-2 deficiency impairs brain growth and promotes tau phosphorylation. J Neurosci 2003;23:7084-7092.

26 Goedert M, Spillantini MG, Jakes R, Rutherford D, Crowther RA: Multiple isoforms of human microtubuleassociated protein tau: sequences and localization in neurofibrillary tangles of Alzheimer's disease. Neuron 1989;3:519-526.

27 Alonso AC, Grundke-Iqbal I, Iqbal K: Alzheimer's disease hyperphosphorylated tau sequesters normal tau into tangles of filaments and disassembles microtubules. Nat Med 1996;2:783-787. Mandelkow EM, Mandelkow E: Tau in Alzheimer's disease. Trends Cell Bio 1998;8:425-427.

Ballatore C, Lee VM, Trojanowski JQ: Tau-mediated neurodegeneration in Alzheimer's disease and related disorders. Nat Rev Neurosci 2007;8:663-672.

30 Ferrer I, Gomez-Isla T, Puig B, Freixes M, Ribé E, Dalfó E, Avila J: Current advances on different kinases involved in tau phosphorylation, and implications in Alzheimer's disease and tauopathies. Curr Alzheimer Res 2005;2:3-18.

-31 Eldar-Finkelman H: Glycogen synthase kinase 3: an emerging therapeutic target. Trends Mol Med 2002;8:126-132.

32 Schechter R, Beju D, Miller KE: The effect of insulin deficiency on tau and neurofilament in the insulin knockout mouse. Biochem Biophys Res Commun 2005;334:979-986.

-33 Liu F, Iqbal K, Grundke-Iqbal I, Hart GW, Gong CX: O-GlcNAcylation regulates phosphorylation of tau: a mechanism involved in Alzheimer's disease. Proc Natl Acad Sci USA 2004;101:10804-10809. 


\section{Errata}

$* * *$

In the article by Peng et al., entitled "Hyperphosphorylation of Tau Protein in Hippocampus of Central Insulin-Resistant Rats is Associated with Cognitive Impairment" [Cell Physiol Biochem 2013;32:1417-1425 (DOI: 10.1159/000356579)], is a printing error in the affiliations. The corrected authors and their affiliations are stated correctly here.

Dantao Peng, ${ }^{\mathrm{a}, \mathrm{f}} \quad$ Xiuqing Pan ${ }^{\mathrm{b}, \mathrm{f}} \quad \mathrm{Ju}_{\mathrm{Cui}}{ }^{\mathrm{c}} \quad$ Yanling Li $^{\mathrm{d}} \quad$ Yueqin Ren $^{\mathrm{e}} \quad$ Jiewen Zhang $^{\mathrm{b}}$

a Department of Neurology, Beijing Hospital, Ministry of Health, Beijing, 'bepartment of Neurology, The People's Hospital of Zhengzhou University, Zhengzhou, 'Beijing Institute of Geriatrics, Beijing Hospital, Ministry of Health, Beijing, ${ }^{d}$ Department of Neurology, Zhengzhou First People's Hospital, Zhengzhou, ${ }^{\text {eThe }}$ Second Affiliated Hospital of Henan Traditional Chinese Medicine University, Zhengzhou, China; ${ }^{\mathrm{f}}$ Equally contributed to this work 